\title{
Evidence for an Opportunistic and Endophytic Lifestyle of the Bursaphelenchus xylophilus-Associated Bacteria Serratia marcescens PWN146 Isolated from Wilting Pinus pinaster
}

\author{
Cláudia S. L. Vicente ${ }^{1,2}$ - Francisco X. Nascimento ${ }^{1,3}$ - Pedro Barbosa ${ }^{1}$. \\ Huei-Mien Ke ${ }^{4,5}$ • Isheng J. Tsai ${ }^{4}$. Tomonori Hirao ${ }^{6}$ • Peter J. A. Cock ${ }^{7}$. \\ Taisei Kikuchi $^{8} \cdot$ Koichi Hasegawa $^{2} \cdot$ Manuel Mota $^{1,9}$
}

Received: 26 May 2016 / Accepted: 8 July 2016 / Published online: 26 July 2016

(C) Springer Science+Business Media New York 2016

\begin{abstract}
Pine wilt disease (PWD) results from the interaction of three elements: the pathogenic nematode, Bursaphelenchus xylophilus; the insect-vector, Monochamus sp.; and the host tree, mostly Pinus species. Bacteria isolated from B. xylophilus may be a fourth element in this complex disease. However, the precise role of bacteria in this interaction is unclear as both plant-beneficial and as plant-pathogenic bacteria may be associated with PWD. Using whole genome sequencing and phenotypic characterization, we were able to investigate in more detail the genetic repertoire of Serratia marcescens PWN146, a bacterium associated with $B$. xylophilus. We show clear evidence that $S$. marcescens PWN146 is able to withstand and colonize the plant environment, without having any deleterious effects towards a susceptible host (Pinus thunbergii), B. xylophilus nor to the nematode model $C$. elegans. This bacterium is able to tolerate growth in presence of xenobiotic/organic compounds, and use phenylacetic acid as carbon source. Furthermore, we pres-
\end{abstract}

Electronic supplementary material The online version of this article (doi:10.1007/s00248-016-0820-y) contains supplementary material, which is available to authorized users.

Cláudia S. L. Vicente

cvicente@uevora.pt

1 NemaLab/ICAAM - Institute of Mediterranean Agricultural and Environmental Sciences, Biology Department, University of Évora, Évora, Portugal

2 Department of Environmental Biology, Chubu University, Kasugai, Japan

3 Departamento de Microbiologia, Laboratório de Microbiologia do Solo, Universidade Federal de Santa Catarina, Florianópolis, Brazil

4 Biodiversity Research Center, Academia Sinica, Taipei, Taiwan ent a detailed list of $S$. marcescens PWN146 potentials to interfere with plant metabolism via hormonal pathways and/ or nutritional acquisition, and to be competitive against other bacteria and/or fungi in terms of resource acquisition or production of antimicrobial compounds. Further investigation is required to understand the role of bacteria in PWD. We have now reinforced the theory that $B$. xylophilus-associated bacteria may have a plant origin.

Keywords Bursaphelenchus xylophilus $\cdot$ Endophyte Nematode $\cdot$ Serratia marcescens $\cdot$ Pine wilt disease

\section{Introduction}

Pine wilt disease (PWD) is considered to be the most devastating disease of Eurasian coniferous forests [1]. At least three major elements are involved in the development of PWD: the
$5 \quad$ Ph.D. Program in Microbial Genomics, National Chung Hsing University and Academia Sinica, Taichung, Taiwan

6 Forest Tree Breeding Center, Forestry and Forest Products Research Institute, Ibaraki, Japan

7 Information and Computer Sciences group, The James Hutton Institute, Invergowrie, DD2 5DA Dundee, UK

8 Division of Parasitology, Faculty of Medicine, University of Miyazaki, Miyazaki, Japan

9 Departamento de Ciências da Vida, Universidade Lusófona de Humanidades e Tecnologias, Lisbon, Portugal 
plant parasitic nematode Bursaphelenchus xylophilus (PWN, pine wood nematode) which is the causal agent of the disease; the wood-boring beetle Monochamus sp. (Cerambycidae) which is the main insect-vector of the PWN; and the host tree which are most Pinus species $[1,2]$. Other organisms have also been shown to be involved in this complex disease, including fungi and bacteria $[3,4]$. In the particular case of bacteria, several studies have reported their dual role in PWD development, as mutualistically associated $[5,6]$ and even putatively synergetic with PWN infection $[1,7,8]$, or as potential host defenders against PWN $[9,10]$. The bacterial communities associated with B. xylophilus are predominantly dominated by $\beta$ - and $\gamma$-proteobacteria, of which Pseudomonas, Burkholderia, Serratia are major representatives [11, 12]. Vicente and co-workers [11] compared the culturable bacterial communities of PWN obtained from long-term lab cultures and from symptomatic Pinus pinaster and found that Serratia was highly abundant in both. In the characterization of bacterial communities of Monochamus galloprovincialis, the key insect-vector of PWN in European forests, a high abundance of Serratia sp. was also reported [7].

The genus Serratia is known for its environmental adaptability, and is easily found as a free-living or hostopportunistic microorganisms in water, soil, animals, plants, and even insects [13]. Eighteen species and four subspecies of Serratia have been described to date [14], and almost all of these have been, or in the process of, being genome sequenced. The type species of this genus is $S$. marcescens and is commonly identified as a multidrug-resistant nosocomial pathogen [15]. This species also has been isolated as a pathogen from insects, $S$. marcescens subspecies marcescens Db11 [16], from plants, S. marcescens AGPim1A [17], and as a non-pathogenic associated bacterium from PWN, formerly referred as Serratia sp. PWN146 [7, 18]. Phenotypically, S. marcescens PWN146 is characterized by: multidrugresistance $\left(\mathrm{Amp}_{50}, \mathrm{Ery}_{50}, \mathrm{Kan}_{50}, \mathrm{Tet}_{15}, \mathrm{Rif}_{50}, \mu \mathrm{g} / \mathrm{mL}\right)$; cellulase activity; formation of biofilm; production of siderophores and by its ability to induce hypersensitive reactions (HR) in Nicotiana tabacum [18]. Moreover, S. marcescens PWN146 has no ACC deaminase activity, phosphate solubilization, and does not significantly promote root elongation of Brassica campestris, but tested positively for indole acetic acid (IAA) and exopolysaccharides (EPS) production [18]. Vicente et al. [7] showed that Serratia marcescens PWN146, and two more Serratia sp. (LCN4 and LCN16), could assist PWN in severe oxidative stress conditions, suggesting a facultative association with the nematode during PWD progression. Hence, in this study, we aimed to: (1) obtain the whole genome sequence of $S$. marcescens PWN146 in order to gain new insights into the genetic content that could explain PWN146 behavior in a plant-associated lifestyle and ultimately in interaction with the PWN; (2) prove $S$. marcescens PWN146 ability to colonize plants; and (3) to complete phenotypic characterization with biochemical description, nematicidal activity against B. xylophilus and the model C. elegans, and pathogenicity in a susceptible host. Understanding the host-microbe interactions can be exploited for the development of new strategies of management and control of PWD.

\section{Experimental Procedures}

\section{Bacteria Growth and Biochemical Characterization}

Non-pigmented S. marcescens PWN146 was originally isolated from the cuticle of PWN extracted from PWDsymptomatic $P$. pinaster [12]. Unless otherwise stated, S. marcescens PWN146 was grown in Luria Broth (LB) medium at $28{ }^{\circ} \mathrm{C}$, washed with $1 \times$ PBS (phosphate-buffered saline), and $\mathrm{OD}_{600}$ adjusted to $0.5-0.8$. The biochemical characterization of this isolate was conducted using the VITEK 2 system with GN (Gram-negative) cards. Tolerance and degradation of terpenes and aromatic compounds were tested by inoculation of $20 \mu \mathrm{L}$ of PWN146 overnight culture in, respectively, trypticase soy broth (TSB) and minimal medium M9 (without $\mathrm{C}$ source), and incubation at $28{ }^{\circ} \mathrm{C}$ during 5 days. The following compounds were analyzed: phenol $(50 ; 100$; $200 \mathrm{mg} / \mathrm{L})$, benzoic acid $(10 ; 50 ; 100 \mathrm{mg} / \mathrm{L})$, xylol $(0.1,0.5$, $1 \%)$, toluene $(0.1,0.5,1 \%),(+)-\alpha$-pinene $(0.1,0.5,1 \%)$, $(-)-\alpha$-pinene $(0.1,0.5,1 \%), \alpha$-pinene (isomer mix) $(0.1,0.5$, $1 \%),(+)-\beta$-pinene $(0.1,0.5,1 \%)(-)-\beta$-pinene $(0.1,0.5$, $1 \%), \beta$-pinene (isomer mix) $(0.1,0.5,1 \%),(+)-3$-carene $(0.1,0.5,1 \%), 3$-carene $(0.1,0.5,1 \%)$ and $\mathrm{R}-(+)$-limonene $(0.1,0.5,1 \%)$.

\section{Nematicidal Activity Against $C$. elegans and B. xylophilus Ka4}

Nematicidal activity of PWN146 was tested against Caenorhabditis elegans and B. xylophilus. C. elegans N2 Bristol strain culturing and handling were carried out at $20{ }^{\circ} \mathrm{C}$ as described by Brenner [19]. Synchronized L1 stage C. elegans were obtained by treating egg-containing/or gravid adults with $\mathrm{NaOCl}$ and allow to grow on NGM plates seeded with E. coli OP50 (control) or S. marcescens PWN146 at $20{ }^{\circ} \mathrm{C}$, for 24,48 , and $72 \mathrm{~h}$. At each time-point, C. elegans were removed from the plate, washed with M9 buffer (three times) to remove bacterial cells, and picked for microscope slide preparation. Agar $(5 \%, w / v)$ pads were prepared containing 20-25 nematodes. Images were taken using a Nikon SMZ1000 binocular microscope equipped with a Visualix VTCH-1.4CICE CCD camera. The deleterious effects of S. marcescens PWN146 in C. elegans N2 development was assessed by measuring the nematode size at each time-point $(n=20-25)$. Image processing was conducted using Image $\mathrm{J}$ 
[20]. Data was statistically analyzed using STATISTICA software version 7.0. Homogeneity of variances was checked by Levene's test. Data was subjected to ANOVA analysis to statistical differences between treatments (OP50 and PWN146) each time $(24,48$, and $72 \mathrm{~h})$. A post-hoc Tukey's test at $95 \%$ confidence level was used for multiple means comparison after significant ANOVA.

B. xylophilus $\mathrm{Ka} 4$, previously grown in $B$. cinerea on barley seeds, were extracted overnight in Baermann funnels, surface-sterilized with L-lactic acid $(3 \%, v / v)[21]$ and suspended in sterile $1 \times$ PBS at a concentration of 1.5 nematodes per $\mu \mathrm{L}$. Two treatments were established: (1) $100 \mu \mathrm{L} 1 \times$ PBS $+100 \mu \mathrm{L}$ Ka4 suspension (approximately 150 nematodes) as null treatment; and (2) $100 \mu \mathrm{L}$ of $\mathrm{Ka} 4$ suspension (approximately 150 nematodes) and $100 \mu \mathrm{L}$ of bacterial suspension. This experiment was established in a sterile 96-well plate whereas each treatment was repeated three independent times with five repetitions. The plate was incubated at $25^{\circ} \mathrm{C}$, and nematode mortality was checked $24 \mathrm{~h}$ later as described by Barbosa et al. [22].

\section{Pathogenicity in Pinus thunbergii}

The pathogenicity of $S$. marcescens PWN146, alone or in association with B. xylophilus Ka4, was tested in 3.5-yearold greenhouse grown $P$. thunbergii saplings. The saplings were grafts obtained from a single pine tree at the Forest Products Research Institute, Forest Tree Breeding Center in Ibaraki, Japan in 2012. Four treatments, each with four biological replicates totally randomized, were established: (1) $P$. thunbergii (inoculation with sterile $\mathrm{dd}_{2} \mathrm{O}$ ); (2) $P$. thunbergii inoculated with $B$. xylophilus Ka4; (3) $P$. thunbergii inoculated with $S$. marcescens PWN146; and (4) P. thunbergii inoculated with B. xylophilus $\mathrm{Ka} 4$ in association with $S$. marcescens PWN146. Both nematodes and bacteria were prepared as mentioned above. Nematode inoculum was adjusted to 1000 nematodes (mixed-stages) per $100 \mu \mathrm{L}$ of $\mathrm{ddH}_{2} \mathrm{O}$. The inoculation procedure was conducted according to Futai and Furuno [23]. Two small-wounds $(1 \mathrm{~cm})$ were made in the middle of stem in the first and second nodes using a sterile blade. A sterilized piece of cotton was placed in the wound site and fixed with parafilm ${ }^{\mathrm{TM}}$ (Bemis Company, Inc.). Nematodes suspension was directly injected into the cotton (inoculation point). The trial was maintained in the greenhouse conditions for 10 weeks (between 16th July 2015 till 28th August 2015). Observations were conducted every week, and watering was conducted twice a week. Symptomology was scored as follows: 0 , no needle discoloration; 1, $25 \%$ needle discoloration (brown yellowish); 2, $50 \%$ needle discoloration (yellowish to brown); 3, $75 \%$ needle discoloration (needles browning); and 4, $100 \%$ needle discoloration (complete wilted tree). The disease incidence was calculated according to Fang [24] (Equation 1):

Disease incidence (\%)

$$
\begin{aligned}
= & \frac{\Sigma \text { number of disease plants } \times \text { symptom stage }}{\text { total number of plants } \times \text { highest symptom stage }} \\
& \times 100
\end{aligned}
$$

\section{Genome Sequencing, Annotation, and Comparative Analysis}

A single-colony culture of $S$. marcescens PWN146 in $10 \mathrm{~mL}$ of LB was incubated overnight shaking at $27{ }^{\circ} \mathrm{C}$. Genomic DNA was then extracted using the QIAGEN Genomic DNA Purification kit (first and second sequencing round) and QIAGEN Genomic-tip 500/G kit (third sequencing round), following the manufacturer's instructions.

The DNA was first sequenced on the Roche Titanium 454 platform at the Centre for Genomic Research, University of Liverpool, with large-insert 3-kb paired-end libraries. This gave a total 329,241 sequences, mean trimmed length $425.6 \mathrm{bp}$, totalling $140 \mathrm{Mbp}$. Initial assemblies were performed with Roche "Newbler" gsAssembler [25], and MIRA v4.0.2 [26]. This data was supplemented with two runs from Illumina MiSeq commissioning test runs at the James Hutton Institute, as one of 11 and 4 barcoded samples, yielding 1.3 and 5.7 million paired reads respectively, totalling $1.8 \mathrm{Gbp}$. Again, multiple assemblies were evaluated, and while improved, a closed chromosome was not achieved. In the third and final round of sequencing, one $20-\mathrm{kb}$ insert SMRTbell library was generated with size selection on the BluePippin (Sage Science). The S. marcescens PWN146 genome was sequenced in 2 SMRT P6-C4 chemistry cells on the PacBio RS II platform (Pacific Biosciences), generating 66,159 reads (N50 16,506 bp) totaling $844.9 \mathrm{Mb}$. The Pacbio sequences were assembled using the Celera assembler PBcR hierarchical pipeline (with default parameters) [27] and corrected using Quiver algorithm [28], giving a closed chromosome and two closed plasmids.

Nucleotide sequence position 1 of $S$. marcescens PWN146 genome was ordered according to the published $S$. marcescens Db11 and SM39 [15], and checked using MAUVE 2.4 (Supplemental Fig. 1) [29]. Automatic genome annotation was performed using PROKKA 1.11 [30], further supported by BLAST2GO [31] and KAAS (KEGG Automatic Annotation Server) [32]. Genome visualization and manual review was performed in ARTEMIS 16.0 [33].

BLAST Ring Image Generator (BRIG) [34] was used for genome comparison between $S$. marcescens PWN146 and other complete genomes of $S$. marcescens (Table 1), and 
Table 1 List of complete genomes of $S$. marcescens from environmental and clinical sources

\begin{tabular}{|c|c|c|c|c|c|c|c|}
\hline Strain & Origin & Size $(\mathrm{Mb})$ & $\mathrm{GC} \%$ & Genes & CDS & Replicons & Reference \\
\hline Serratia marcescens PWN146 & Environmental & 5.4 & 59.28 & 5405 & 5068 & Chromosome 2 plasmids & This study \\
\hline Serratia marcescens subsp. marcescens Db11 & Clinical & 5.1 & 59.50 & 4743 & 4607 & NZ_HG326223.1 & [35] \\
\hline Serratia marcescens FGI94 & Environmental & 4.8 & 58.90 & 4436 & 4290 & NC_020064.1 & {$[36]$} \\
\hline Serratia marcescens WW4 & Environmental & 5.2 & 59.59 & 4871 & 4744 & $\begin{array}{l}\text { NC_020211.1 } \\
1 \text { plasmid }\end{array}$ & {$[37]$} \\
\hline Serratia marcescens SM39 & Clinical & 5.3 & 59.73 & 4950 & 4806 & $\begin{array}{l}\text { NZ_AP013063.1 } \\
2 \text { plasmids }\end{array}$ & [15] \\
\hline Serratia marcescens CAV1492 & Clinical & 5.8 & 58.63 & 5460 & 5274 & $\begin{array}{l}\text { NC_CP011642.1 } \\
6 \text { plasmids }\end{array}$ & {$[35]$} \\
\hline Serratia marcescens RSC-14 & Environmental & 5.1 & 59.60 & 4745 & 4593 & СР012639.1 & {$[35]$} \\
\hline Serratia marcescens SmUNAM836 & Clinical & 5.2 & 59.82 & 4944 & 4774 & $\begin{array}{l}\text { CP012685.1 } \\
1 \text { plasmid }\end{array}$ & {$[38]$} \\
\hline
\end{tabular}

Data from Serratia marcescens strains CAV1492, Db11, WW4, SM39, FGI94, RSC-14, and SmUNAM836 retrieved from NCBI, Genome Assembly and Annotation Report (http://www.ncbi.nlm.nih.gov/genome/genomes/1112?)

genomic islands were annotated by Island Viewer 3.0 using the available genomes as reference: $S$. marcescens Db11, S. marcescens CAV1492, S. marcescens WW4, S. marcescens SM39, and S. marcescens FGI94 [39]. The average nucleotide identity (ANI) and tetranucleotide signature was calculated using JSpecies 1.2.1 software [40]. Orthologous genes analysis between $S$. marcescens genomes (Table 1) and $S$. marcescens PWN146 was conducted using OrthoFinder 0.4 [41]. For phylogenetic analysis of PWN146, four housekeeping genes ( $a t p D, d n a J, g y r B$, and $r p o B$ ) of Serratia-type strains were concatenated using Seaview 4.0 [42], and maximum likelihood (ML) tree was constructed using K2 (Kimura 2-parameters) in MEGA6 [43]. Phylogenetic robustness was inferred by bootstrap analysis using 1000 iterations.

\section{Scanning Electron and Confocal Laser Scanning Microscopy}

To observe $S$. marcescens PWN146 adhesion to B. xylophilus $\mathrm{Ka} 4$ cuticle after 1 and $48 \mathrm{~h}$ contact, nematodes were firstly surface-cleaned with L-lactic acid $(3 \%, v / v)$ and suspended in sterile $\mathrm{ddH}_{2} \mathrm{O}$. After 1 and $48 \mathrm{~h}$ contact, nematodes were removed from bacterial suspension and prepared for scanning electron microscope (SEM) observation as described in Shinya et al. [44]. SEM images were taken using a JSM6510LA (JEOL, Tokyo, Japan).

The ability of $S$. marcescens PWN146 to infect and colonize plants was tested on fast-growing vigor tomato plants (Lycopersicon esculentum cv "Momotaro"). GFP-labeled S. marcescens PWN146 made previously [7] was prepared as follows. Bacteria were grown overnight in LB broth supplemented with $30 \mu \mathrm{g} \mathrm{mL}^{-1}$ gentamicin and $50 \mu \mathrm{g} \mathrm{mL} \mathrm{m}^{-1}$ rifampicin, washed three times with $1 \times \mathrm{PBS}$, and the $\mathrm{OD}_{600}$ adjusted to 0.5 . Tomato seeds were surface-sterilized with $70 \% \mathrm{EtOH}(w / v)$ for $1 \mathrm{~min}$, and $1 \% \mathrm{NaOCl}(w / v)$ for $10 \mathrm{~min}$, followed by several rinses with sterile $\mathrm{ddH}_{2} \mathrm{O}$. Seeds (15-20) were treated with two treatments: (1) $\mathrm{ddH}_{2} \mathrm{O}$ only, considered the null treatment; and (2) bacteria for $1 \mathrm{~h}$. Afterwards, seeds were transferred to fresh agar plates. Ten days after inoculation (DAI), seedling colonization was observed with a confocal laser scanning microscope LSM710 (Carl Zeiss, Germany) equipped with an Argon laser (458, $477,488,514 \mathrm{~nm}$ ) and detectors for GFP (FITC dye, 495$590 \mathrm{~nm}$ ) and plant auto fluorescence (492-510 nm). Images were obtained in a z-series with 20-30 optical sections, and processed in ZEN Image 2.0 software (Carl Zeiss, Germany).

\section{Results and Discussion}

\section{Genome Structure and Comparative Analysis}

The complete genome of $S$. marcescens PWN146 is contained in one single chromosome of 5,485,668 bp with an overall $\mathrm{G}^{+}$ C content of $59.28 \%$, and two F-like plasmids pPWN146.1 $(139,402$ bp with $\mathrm{G}+\mathrm{C}$ of $55,8 \%)$ and pPWN146.2 (61,142 bp with $\mathrm{G}+\mathrm{C}$ of $56.5 \%)$. A total of 5405 genes were predicted in the PWN146 genome (chromosome and plasmids), from which 5288 (97.8\%) were considered coding sequences (CDS) with 1076 (20.3\%) hypothetical proteins of unknown function, and 117 (2.1\%) RNA genes (22 rRNA, 94 tRNA, and 1 tmRNA). Furthermore, 4820 (91.1\%) CDS were annotated with InterPro signatures, 3448 (65.2\%) and $3159(59.7 \%)$ were assigned, respectively, to Gene Ontology (GOs) and KO terms (Supplementary Tables S1 and Fig. S2). Both plasmids, pPWN146.1 and pPWN146.2 have type IV pili (conjugal transfer pilus assembly proteins) which allow their motility [45]. In fact, 
pPWN146.1 has two sets of type IV pili proteins suggesting co-integration of plasmids [46]. Plasmid pPWN146.1 also has toxin-antitoxin systems integrated (vap $C B$, and incomplete relE/stbE-relB/stbD), which are involved in plasmid transfer fitness [47]. As in pPWN146.1, pPWN146.2 is mostly composed by hypothetical proteins of unknown function, which limits the interpretation of their role.

Bacterial genomes with low number of MGEs (mobile genetic elements), such as phages, transposons (Tn) or inserted elements (IS), are usually found in bacteria adapted to restricted environments, such as obligate intracellular symbionts [48]. In the PWN146 chromosome, two Tn10 transposon sequences (Tn10-Tet, tetracycline resistance), 20 transposases/ IS and 89 phage-like proteins were found, mostly surrounding hypothetical proteins. PWN146 chromosome also contains Clustered Regularly Interspaced Short Palindromic Repeats (CRISPR) Type I (universal cas1, PWN146_04951; cas3, PWN146_04952), subtype I-F (csy1234, PWN146_0495356), flanked by two repeat regions. CRISPR_Cas systems are known to provide adaptive and hereditary immunity against previously encountered bacteriophages and plasmids, and can be located in specialized genome regions encoding defense or stress proteins [49]. PWN146 CRISPR_Cas is located upstream of a phosphonate $p h n$ operon ( $p h n P O N M L K J I H G F)$ reported to be involved in the acquisition of phosphorus from the natural environment under phosphate limiting condition, and also linked with antibiotic activities or components of cellular macromolecules (i.e., glycoproteins or phospholipids) [50].

Comparative genome analysis between all $S$. marcescens strains (Table 1) is presented in Fig. 1a. The closest Serratia strains to PWN146 are the host-pathogen $S$. marcescens CAV1492, SmUNAM836 and SM39 (Figs. 1a and 2a). The ANI between PWN146 and CAV1492 and SmUNAM836 was $98 \%$, followed by SM39 and Db11 (96 \%), and environmental strains WW4 and RSC-14 (95\%). All strains belong to the same species according to the $95-96 \%$ range limits for species definition [40]. The tetranucleotide signature between PWN146 and CAV1492 was 0.9994 (Fig. 2a). These results are further corroborated by the phylogenetic analysis of housekeeping genes (atpD, dnaJ, gyrB, and rpoB) (Fig. 2b), which cluster PWN146 jointly with CAV1492 within the $S$. marcescens clade. The environmental strain $S$. marcescens FGI94, an insect isolate, was considered the most different strain to PWN146 with an ANI of $86 \%$, actually suggesting this is a different Serratia species. A total of 16 genomic islands (GIs), ranging between 4 and $26 \mathrm{~kb}$, were predicted in the PWN146 genome by at least one method (SIGI-HMM, IslandPath-DIMOB, IslandPick) (Fig. 1b, Supplemental Table S2) [39]. These GIs include 258 genes, which are mostly annotated as hypothetical proteins and MGEs, and genes involved in the biosynthesis of antibiotics (gramicidin, igr $B$, PWN146 00556, PWN146 00573 and PWN146 00251; tyrocidine, tycC, PWN146 00554 and PWN146 00571; plipastatin, ppsDE, PWN146_ 00557 and PWN146_00574; and surfactin, srfAD, PWN146_00575); drug resistance proteins (bicyclomycin resistance protein, bsr, PWN146_03395); cell wall-degrading enzymes (chitinase class I, PWN146_02145); and insecticidal toxin (type-1Aa cytolytic delta-endotoxin, cyt1Aa, PWN146_05139). Curiously, cyt1Aa is surrounded by IS66 (PWN146_05136-38) and IS2 (PWN146_05140), which may suggest a horizontal genetransfer (HGT) event, perhaps from the animal and human pathogen Aeromonas salmonicida (BlastP: $49 \%$ identity, $e$ value $\left.5 e^{-60}\right)$ [51]. Aeromonas sp. were already isolated from B. xylophilus extracted from PWD-symptomatic pines as well as B. mucronatus $[10,52]$. Orthologous analysis predicted 276 CDS unique to PWN146 in comparison with the other S. marcescens strains (Table 1), most of which were found in the PWN146 predicted GIs (Supplemental Table S2). Furthermore, the same analysis performed between PWN146 and the closest $S$. marcescens CAV1492, SmUNAM836 and SM39 (Fig. 2c) showed that the number of conserved genes in PWN146 ranged between 4302 and 4327 and the number of unique genes varies between 740 and 763 genes. Among the unique genes of PWN146 in relation to CAV1492, we identified genes predicted in plant-associated bacteria, such as: plant cell wall-degrading enzymes, cellulase (PWN146_01039) and alpha-beta hydrolases (PWN146_02529); and xenobiotic efflux pumps (aaeAB, PWN146_00522, PWN146_00524; PWN146_05144 and PWN146_05146) [53, 54].

\section{Nematode-Bacteria Interaction}

The nematicidal activity of $S$. marcescens PWN146 was tested against C. elegans (Fig. 3) and the PWN B. xylophilus Ka4. The development of $C$. elegans was slightly delayed in the presence of PWN146 after $48 \mathrm{~h}$ and $72 \mathrm{~h}$ incubation compared with E. coli OP50. C. elegans is a simple model to test virulence mechanisms of diverse bacteria, from pathogens to symbionts [55, 56]. In the case of B. xylophilus Ka4, PWN146 showed no nematicidal effect after $24 \mathrm{~h}$ (Supplemental Table S3). These results indicate that $S$. marcescens PWN146 has no nematicidal effect towards nematodes, and are consistent with previous observations that suggested a beneficial effect towards different $B$. xylophilus strains under specific conditions [7]. In contrast, Paiva et al. [9] reported that another B. xylophilus-associated bacteria Serratia sp. A88copa13 was highly toxic to the nematode and attributed this activity to the presence of secreted serine proteases and other bacteriocins. The methodology used in the present study was different from Paiva et al. [9], making it difficult to compare directly with this study. However, we could also find three serine proteases from subtilisins family (MEROPS S08.094) (PWN146_01829, PWN146_04255, and 


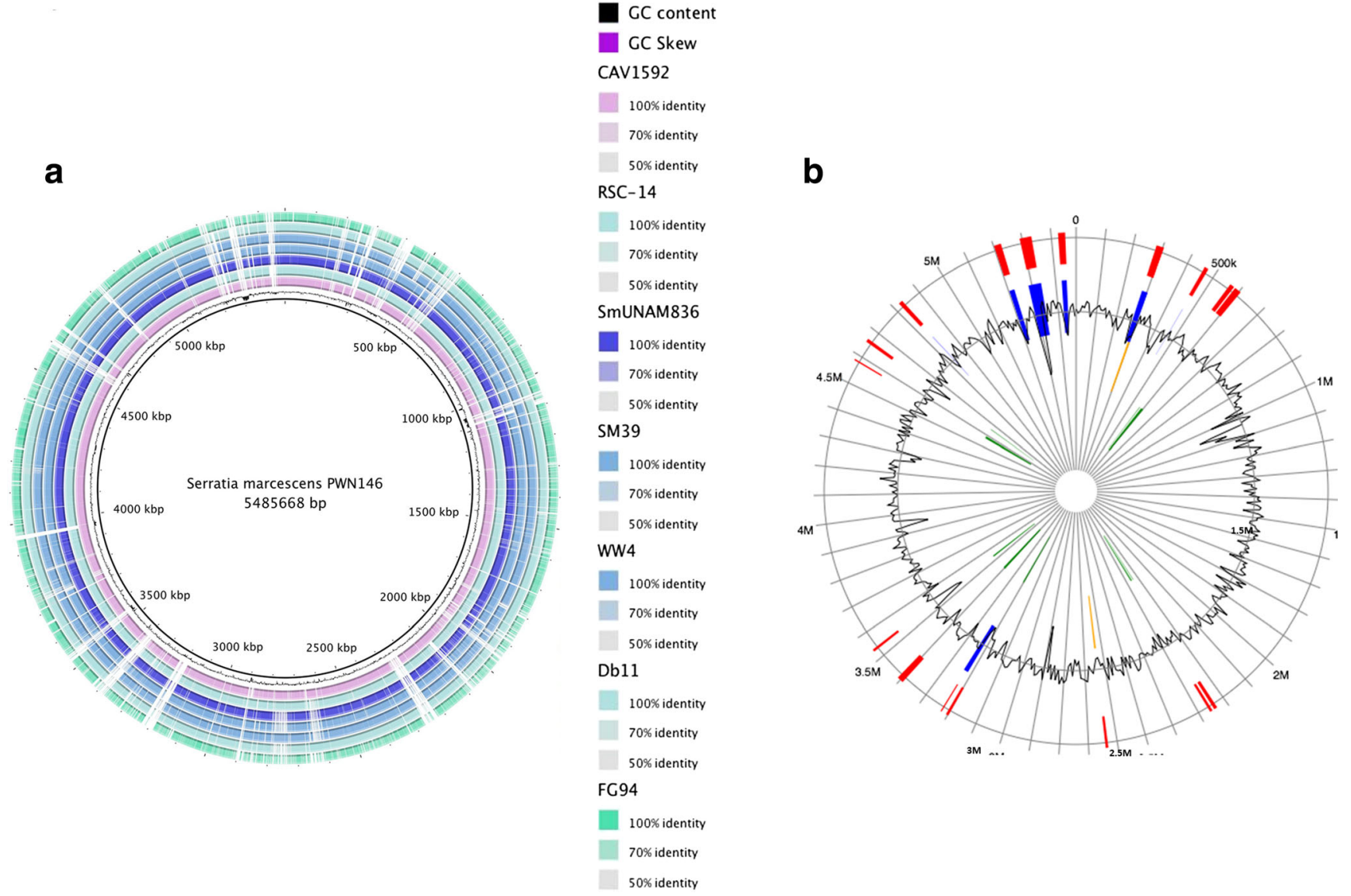

Fig. 1 Circular visualization of comparative genome analysis of $S$. marcescens strains. This analysis was performed in BRIG (BLAST Ring Image Generator) [34] (a); and in IslandViewer 3.0 [39] for genomic Islands (GIs) prediction (b). GIs in blue, orange and green indicate, respectively, prediction by IslandPath-DIMOB, SIGI-HMM, and IslandPick approaches. GIs in red were predicted by at least one of the three above described approaches

bacteria that show phenotypic plasticity, that are able to survive and colonize the plant environment and, under certain circumstances, may be nematode-synergetic [7, 10, 58]. GFP-tagged S. marcescens PWN146 was inoculated in tomato seeds and observed for their ability to colonize and invade the root system. After 10 DAI, we were able to detect GFP-PWN146 attached to the root hairs (Fig. 4c) and within, occupying the intercellular spaces (Fig. 4d). Moreover, the inoculation of S. marcescens PWN146 alone has not induced PWNsymptoms in 4-year-old $P$. thunbergii during $10 \mathrm{WAI}$ (weeks after inoculation) (Supplemental Fig. 4). This result is in contradiction with previous results [18]. S. marcescens PWN146 have induced disease symptoms in 1-year-old $P$. pinaster even though not as B. xylophilus [18]. This result may suggest the different hosts and development age can be accounted for the different behaviors of $S$. marcescens PWN146. In this trial, the first PWD symptoms were seen in the treatment $B$. xylophilus Ka4 around the second and third WAI, while in the treatments B. xylophilus $\mathrm{Ka} 4+S$. marcescens PWN146 the symptoms appeared the third and fourth WAI. Pine trees from both treatments were wilted between the ninth and tenth WAI. 
a
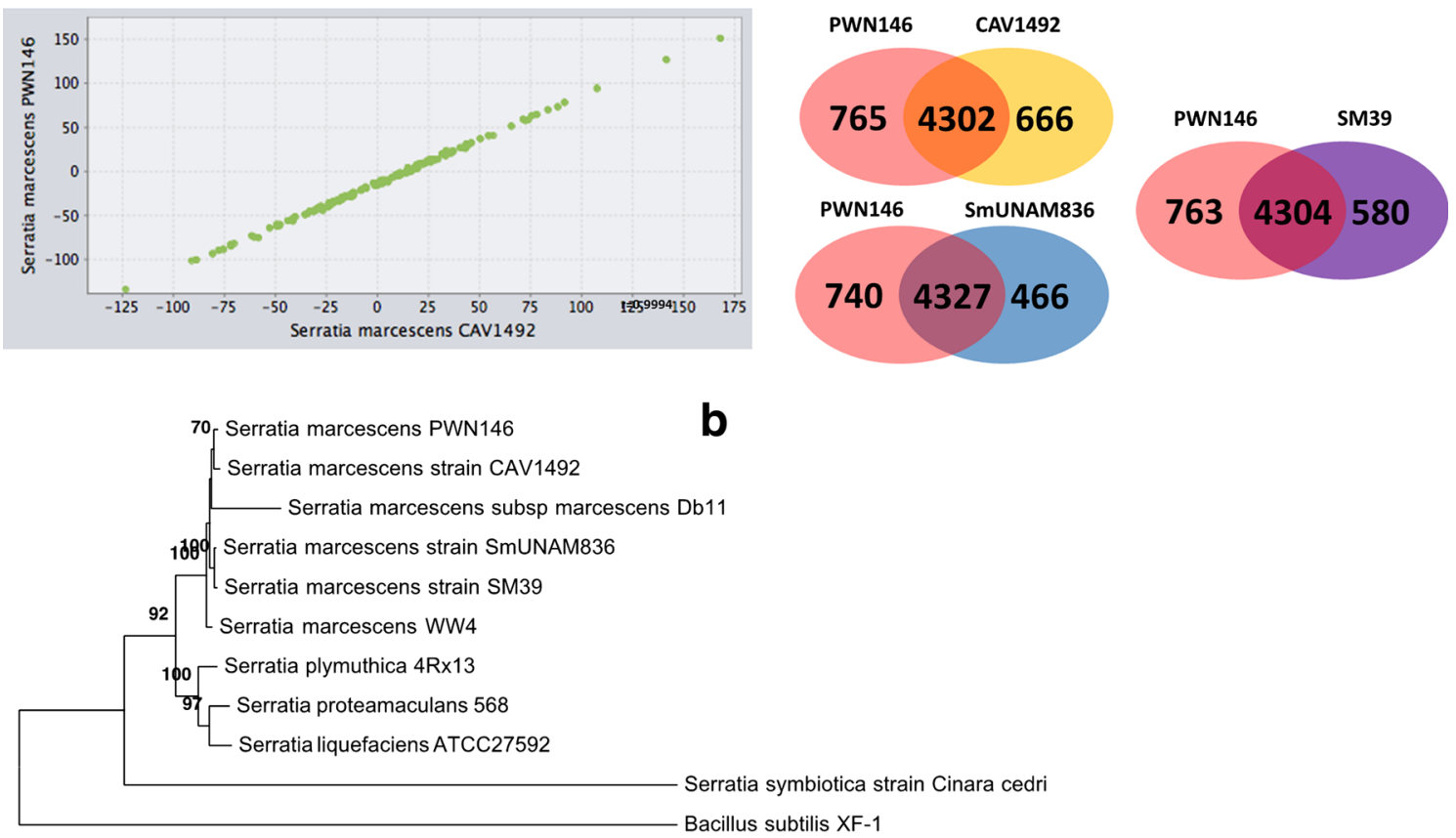

Bacillus subtilis XF-1

0.1

Fig. 2 Taxonomic inference of $S$. marcescens PWN146 and orthologous analysis between the closest $S$. marcescens (Table 1). The tetranucleotide signature between PWN146 and CAV1492 is shown in a. The

In view of the previous results, PWN146 genome analysis was focused on understanding its genetic repertoire which could facilitate a plant-associated lifestyle (Supplemental Table S3). Using comparative genomics, Mitter et al. [48] reported the existence of typical endophytic traits among plantassociated bacteria; these traits were not exclusively linked to the endophytic behavior, but based on different interaction strategies with the host plant. Successful endophytes, facultative or obligate, express colonization traits that allow their entrance in the plant habitat. These steps involve: recognition,

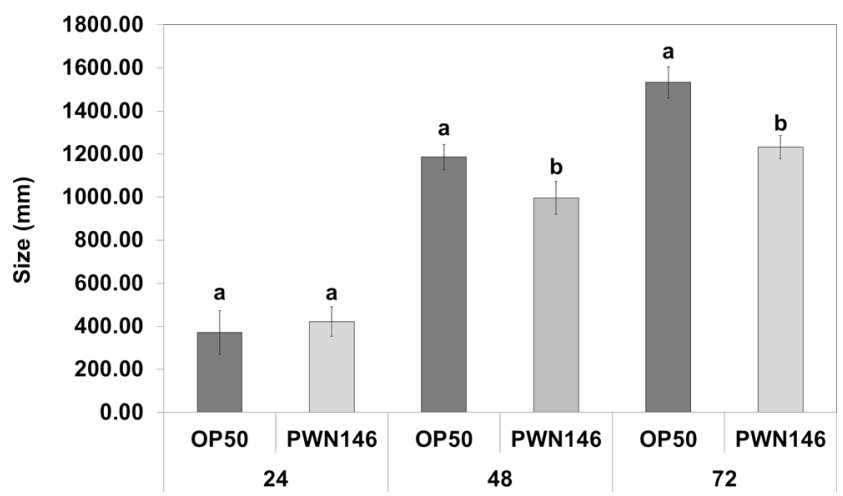

Fig. 3 Growth effect of $S$. marcescens PWN146 in the nematode model $C$. elegans during 24, 48 and $72 \mathrm{~h}$. The $E$. coli strain OP50, routinely used for C. elegans growth, was used to infer the normal nematode growth. Error bars indicate standard deviation. Different letters indicate statistical differences at $95 \%$ confidence level (post-hoc Tukey's Test) phylogenetic analysis of housekeeping genes is presented in $\mathbf{b}$. Venn diagrams showing the orthologous between PWN146 and CAV1492, SmUNAM836, SM39 are indicated in $\mathbf{c}$

adherence, invasion, colonization, and growth [59]. The $S$. marcescens PWN146 genome encode genes potentially involved in these first steps of plant-bacteria interaction. PWN146 is motile, with a complete flagella biosynthesis set (PWN146_02410-53) and chemotaxis proteins such as methylaccepting chemotaxis proteins (i.e., $m c p$, PWN146_00924; tsr, PWN146_01795, PWN146_02459, PWN146_04878; tar, PWN146_04546; tap, PWN146_02458, PWN146_04818), chemotaxis protein MotAB (PWN146 02462-63), and the two-component system cheZYBRWA (PWN146 02454-57, PWN146_02460-61). Surface adhesion in PWN146 may be accomplished by means of fimbria adhesins ( $m r k D$, PWN146_00970, PWN146_02613), surfactin (PWN146_00575), filamentous hemagglutinins (fhaB, PWN146_03175), and the bcsAZC operon for cellulose biosynthesis (PWN146 04615-18), which was shown to be related to root adhesion [60]. Its ability to enter the host plant may be passive, through wood cracks, or active by secreting plant cell wall-degrading enzymes [61]. In PWN146, we found one gene that could encode a cellulase (PWN146 01039), four genes coding for alpha-beta hydrolases (PWN146_02229, PWN146_02529, PWN146_02579, PWN146_03420), cupin (PWN146_02588) and an alternative pathway, although incomplete, for degradation of galacturonate via $u x a A B$ (PWN146_02810-11, PWN146_03205) [60].

Several characteristics are considered competitive factors for a plant environment, namely detoxification of reactive 

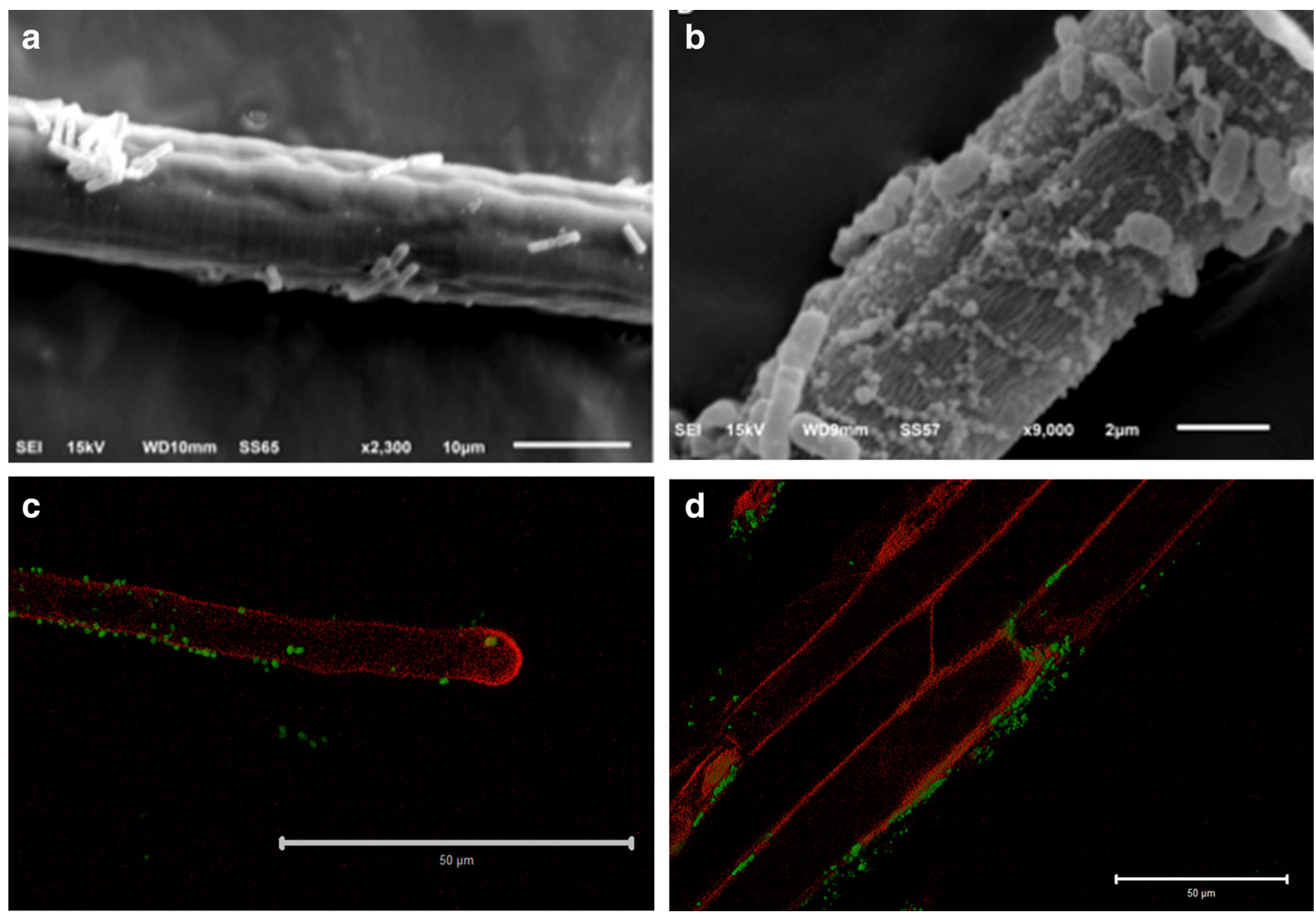

Fig. 4 Microscopic observations of $S$. marcescens PWN146 in interaction with nematode and plant. Images $\mathbf{a}$ and $\mathbf{b}$ show $S$. marcescens PWN146 attachment to B. xylophilus Ka4 cuticle, after $48 \mathrm{~h}$ association, using SEM microscopy. Images $\mathbf{c}$ and $\mathbf{d}$ show,

oxygen species (ROS) and secondary compounds, hormonal stimulation, nutrient acquisition, and production of antimicrobial compounds $[48,59,62,63]$. The oxidative burst is one of the primary defense mechanisms of plants used against abiotic (i.e., climatic conditions) or biotic (i.e., microorganism infection) stresses, and results in the massive production of ROS $\left(\mathrm{O}_{2}^{-}, \mathrm{H}_{2} \mathrm{O}_{2}, \mathrm{OH}^{-}\right)$as a means of barring invasion and network signaling for internal regulation [64]. In planta, endophytic bacteria are well equipped with scavenging enzymes for ROS neutralization, including superoxide dismutases (SODs), catalases (CATs), and peroxiredoxins (PRXs), as well as with extracellular polysaccharides and intracellular polyesters $[65,66]$. Previously, Vicente et al. [7] showed that S. marcescens PWN146 was able to tolerate high concentrations of $\mathrm{H}_{2} \mathrm{O}_{2}$ (up to $100 \mathrm{mM}$ ), thus indicating its resilience under oxidative stress conditions. In S. marcescens PWN146 genome, we found a total of 3 SODs (Mn-SOD encoded by $\operatorname{sod} A$, PWN146 04547; Fe-SOD encoded by $\operatorname{sod} B$, PWN146 01640; and Cu-Zn-SOD encoded by sodC, PWN146 01653); 2 CATs (katA, PWN146 02860; catalaseperoxidase $k a t G$, PWN146_02711); 1 PRX ( $\alpha$-hydroperoxide

respectively, the colonization of tomato root hairs and internal localization by GFP-labeled $S$. marcescens PWN146, after 10 DAI (days after inoculation)

reductase $a p h D$, PWN146_03228) and more 4 putative PRXs (bcp_1, PWN146_03146; bcp_2, PWN146_03147; PWN146_04298; tsa, PWN146_00357); 1 chloroperoxidases (cpo, PWN146 02709); 1 thiol peroxidases ( $t p x$, PWN146_02051); and 1 glutathione peroxidase ( $g x p$, PWN146_01611). In addition, we also found 5 glutathione S-transferases (GSTs) (gstB1, PWN146_00870; gstB2, PWN146_01664; gstB3, PWN146_02581; gstB4, PWN146_03977; $y f c F$, PWN146_03851); and 5 glutaredoxin (grxA, PWN146_01113; grxD, PWN146_01642; grxB, PWN146_02296; grxC, PWN146_03676; nrdH, PWN146_03429). We also identified organic hydroperoxide resistance proteins (ohrRB, PWN146_04597-98), and two genes encoding paraquat-inducible proteins ( $p q i A B$, PWN146 01204-05). The major oxidative stress and general stress regulons, respectively, OxyR/SoxR and RpoS [67], were found in $S$. marcescens PWN146 (oxyR, PWN146_04297; soxR, PWN146_01236; and rpoS, PWN146 00136). PWN146 also seems capable of detoxifying nitric oxide [68] through nitric oxide dioxygenase ( $\mathrm{hmp}$, PWN146_03325), possibly under regulation by $n r s R$ 
(PWN146_04859), nitric oxide-sensitive transcriptional repressor. Several studies have indicated a relationship between ROS tolerance and siderophore synthesis and iron uptake systems [54, 69]. Wu et al. [54] reported that the regulation of ROS-responsive genes in Pseudomonas putida may be irondependent, since antioxidant enzymes such as catalase and hydroperoxide reductase were surrounded with iron-binding proteins (i.e., bacterioferritins). Similarly, in PWN146, katA is located downstream an $\mathrm{ABC}$ iron complex transport system (PWN146_02857-58) and gpx is surrounded by ABC iron complex proteins (PWN146_01605-07). The putative phytoalexin export system AcrAB multidrug efflux pump (acrBA, PWN146 00495-00496) [60], coupled with acrAB operon repressor ( $a c r R$, PWN146_00497), was also found in PWN146.

The ability of $S$. marcescens PWN146 to grow in the presence of organic compounds, mostly plant-derived aromatic compounds, is shown in Table 2. Although PWN146 could withstand increasing concentrations of almost all compounds tested (except citral, carvacrol, geraniol, 3-eugeniol, and linolool), it could only use phenylacetic acid as a carbon source. In the PWN146 genome, we could find the complete pathways for phenylacetic acid and 4-aminobutyrate (GABA), as seen in other endophytic bacteria [70], but could not find the complete metabolic pathways for limonene and pinene degradation, nor the dit gene cluster involved in diterpenoids degradation [71], which may explain why Serratia sp. PWN146 is unable to use these compounds as its sole carbon source. We were able to identify the most abundant enzymes found in metagenomes of pine beetles [72] involved in the catabolism of limonene and pinene, namely, 3-hydroxyacyl-CoA dehydrogenase/enoylCoA hydratase (PWN146_01252, PWN146_02955, PWN146_04724), two putative genes for limonene 1,2monooxygenase (limB, PWN146_01979; PWN146_04915), 2S-(hydroxymethyl) glutathione dehydrogenase (frmA, PWN146_01004, PWN146_05142) and 1 alcohol dehydrogenase (adhE, PWN146_02193).

Vicente et al. [18] have shown that PWN146 is able to produce the plant auxin IAA and siderophores, but failed to show ACC deaminase activity. Corroborating this, we were not able to find ACC deaminase genes, only $S$-adenosylmethionine synthetase (metK, PWN146 03699) which is involved in the biosynthesis of ethylene [73]. The production of IAA in PWN146 is conducted via indole-3-pyruvate decarboxylase (ipdC, PWN146_03001), the main IAA (IPyA) biosynthesis pathway found in plant-beneficial bacteria [54, 60, 61]. The bacterial volatiles acetoin and 2,3butadineol are also emitted for plant-growth enhancement [74]. Similar to Enterobacter sp. 638 [60], PWN146 genome encodes budABC (budA, acetolactate decarboxylase, PWN146 03021; $b u d B$, acetolactate synthase, PWN146 03022; and $b u d C,(S, S)$ butanediol dehydrogenase, PWN146_01493), and pyruvate dehydrogenase poxB (PWN146 01136), which can also convert small amounts of acetoin. Two genes putatively involved in the catabolism of acetoin and 2,3-butadinediol were found ( $a c o R$, PWN146 01820 and PWN146 03820), however the $a c 0 A B C X$ cluster was not identified [75]. In addition to IAA and volatiles, phytohormone-like substances such as polyamines [63], and pyrroloquinolone quinone [76] have been also considered plantgrowth promoting compounds. PWN146 genome encodes a complete pathway for polyamine biosynthesis, speABDE (PWN146_03697-98; PWN146_03725-26), spermidine/ pustrecine (potDCBA, PWN146_01447-50), and putrescine (potFGHI, PWN146_01117-20) transport systems, and pyrroloquinone quinone operon $p q q E D C B$ (PWN146_01839-42).

Mineral acquisition and supply is also considered a plantgrowth promoting feature of competent endophytes and may include traits such as the production of siderophores for iron sequestration and iron transport systems [59]. Furthermore, siderophores are also considered elicitors of induced systemic resistance (ISR), beneficial in the plant-pathogen interaction [63]. PWN146 is well equipped to compete for iron (Supplemental Table S3) containing 2 ferrous-iron transport systems (feo $A B C$, PWN146_04162-64; efeBOU, PWN146_02401-03), 2 copies of the operon afuCBA, iron (III) transport system (PWN146_00750-52; PWN146_03675-77), ABC transporter Mn/Fe (sitABCD, PWN146_01567-70), seven genes coding for ferric siderophore transporter (tonB), 14 genes coding for iron complex transport system substrate-binding proteins $(f e p B), 12$ genes coding for iron complex transport system permease $(f e p D, f e u B, f h u B)$, and 9 genes coding for iron complex transport system ATP-binding $(f l u C)$. PWN146 also synthesizes enterobactin (entA, PWN146_02687; entF, PWN146_04788; ent $C$, PWN146_02688, PWN146_04790-91; entE, PWN146_04792), which is secreted via entS (PWN146_01900). Siderophores can also be retrieved by biopolymer transport protein (ExbDB, PWN146_03791-92) and the iron can be recovered via enterochelin esterase (fes, PWN146_01903, PWN146_03018, PWN146_04786) [60]. Interestingly, the S. marcescens PWN146 genome also encodes several genes related to rhizosphere competence such as heavy metal and drug transporters [60]. We found genes putatively involved in zinc uptake/ transport (znuACB, PWN146_02267-2269; zntB, PWN146_01974 and PWN146_02044); copper resistance (copA, PWN146_00520; transcriptional factor cueR, PWN146_00526; blue copper oxidase, cueO, PWN146_03723; and copper resistance operon copCD, PWN146_01382-01383); arsenate resistance (ars $C$, PWN146_03160); lead/cadmium/zinc/ mercury-transporting ATPase (zntA, PWN146_04676) and nickel transport protein (nixA, PWN146_00235). PWN146 was able to grow in TSB medium containing $5 \mathrm{mM} \mathrm{CuSO}_{4}$ and $\mathrm{ZnSO}_{4}$ (Table 2). Also, $S$. marcescens PWN146 has the complete operon for urea conversion to ammonia (ureABCEFGD, PWN146 00227-33; utp urea transporter, PWN146 00234; ureR operon transcriptional activator, PWN146_02016), supporting the results from biochemical characterization (Supplemental Table S5). 
Table 2 Serratia marcescens PWN146 growth in increasing concentrations of xenobiotics compounds

\begin{tabular}{|c|c|c|c|c|c|}
\hline \multirow[t]{2}{*}{ Tested compound } & \multicolumn{4}{|c|}{ Growth in TSB } & \multirow{2}{*}{$\begin{array}{l}\text { Growth in M9 } \\
\text { sole carbon source }\end{array}$} \\
\hline & $0.1 \%$ & $0.5 \%$ & $1 \%$ & $1.5 \%$ & \\
\hline (+)- $\alpha$-Pinene & + & + & + & + & - \\
\hline$(-)-\alpha$-Pinene & + & + & + & + & - \\
\hline$\alpha$-Pinene (isomer mix) & + & + & + & + & - \\
\hline$(+)$ - $\beta$-Pinene & + & + & + & + & - \\
\hline$(-)$ - $\beta$-Pinene & + & + & + & + & - \\
\hline (+)-3-Carene & + & + & + & + & - \\
\hline 3-Carene (isomer mix) & + & + & + & + & - \\
\hline$R$-(+)-limonene & + & + & + & + & - \\
\hline$\gamma$-Terpinene & + & + & + & + & - \\
\hline p-Cymene & + & + & + & + & - \\
\hline 2-Undecanone & + & + & + & + & - \\
\hline Mircene & + & + & + & + & - \\
\hline Toluene & + & + & $+/-$ & - & - \\
\hline Xylene & + & + & + & - & - \\
\hline Citral & - & - & - & - & - \\
\hline Carvacrol & - & - & - & - & - \\
\hline Geraniol & - & - & - & - & - \\
\hline 3-Eugenol & - & - & - & - & - \\
\hline \multirow[t]{2}{*}{ Linalool } & - & - & - & - & - \\
\hline & $2.5 \mathrm{mM}$ & $5 \mathrm{mM}$ & $10 \mathrm{mM}$ & $20 \mathrm{mM}$ & \\
\hline $\mathrm{CuSO}_{4}$ & + & + & - & - & n.a \\
\hline \multirow[t]{2}{*}{$\mathrm{ZnSO}_{4}$} & + & + & - & - & n.a \\
\hline & $0.01 \mathrm{mg} / \mathrm{ml}$ & $0.05 \mathrm{mg} / \mathrm{ml}$ & $0.1 \mathrm{mg} / \mathrm{ml}$ & $0.2 \mathrm{mg} / \mathrm{ml}$ & \\
\hline Benzoic acid & + & + & + & + & - \\
\hline Phenol & + & + & + & + & - \\
\hline Phenylacetic acid & + & + & + & + & + \\
\hline
\end{tabular}

List of aromatic compounds and heavy metals tested in TSB and in minimal medium M9 as sole carbon source. Bacterial growth is indicated by $(+)$
Generally, high antibiotic resistance is an intrinsic characteristic of $S$. marcescens [15]. This characteristic was previously seen in PWN146 [18], and is corroborated by the presence of genes encoding for tetracycline, macrolide, beta-lactam, aminoglycoside, fluoroquinolone, chloramphenicol resistance, and the existence of multiple multidrug efflux pumps, such as acrAB (PWN146_00495-96) (Supplemental Table S4). The PWN146 genome also encodes several genes related to the production of antimicrobial compounds, which jointly with antibiotic resistance may suggest a highly fitness and competitive bacterium in its environment. Similar to the endophytic bacteria Pseudomonas fluorescens F113 [77], PWN146 also produces hydrogen cyanide (hcnABC, PWN146_01266-68) and pyoverdine ( $p v c A$, PWN146 00021), considered as potential antimicrobial compounds. Also, as other endophytic bacteria (Azoarcus sp. BH72, Herbaspirillum seropedicae SmR1, Klebsiella pneumoniae 342, Stenotrophomonas maltophilia R551-3, and Enterobacter sp. 638), PWN146 encodes chorismate pyruvate lyase gene (ubiC, PWN146_04006) involved in the catabolism of chorismate into the antimicrobial 4-hydroxybenzoate [63]. Additionally, PWN146 may demonstrate fungal antagonism since it encodes several chitinases in its genome (PWN146_00633, PWN146_02145, PWN146_03120, and PWN146_04604), an ability also reported in the poplar endophyte $\bar{S}$. proteamaculans 568 [78]. Besides the universal two-step secretion systems (SS) Sec and Tat (Twin-arginine translocation), S. marcescens PWN146 encodes type 2 and 6 secretion systems (T2SS and T6SS), mostly characterized for the delivery of toxins and hydrolytic enzymes in both plant-beneficial and plantpathogenic bacteria $[60,79]$. However, it lacks the type three secretion system (T3SS), typically found in plant pathogenic bacteria [24]. We could find several candidate toxins likely to be secreted by all secretion systems, such as: poreforming toxins, hemolysins (tlyC, PWN146 00161; hlyIII, PWN146 03621; shlA, PWN146 04024); and membranedamage toxin, phospholipase $\mathrm{C}^{-}$(plcC, PWN146_03785) (Supplemental Table S4). 
Currently, only four B. xylophilus-associated bacteria have been sequenced, namely, Pseudomonas sp. M47T1 [80], Serratia sp. M24T3 [81], P. fluorescens GcM5-1A [82], and Serratia sp. LCN16 [58]. Pseudomonas sp. M47T1, Serratia sp. M24T3, and Serratia sp. LCN16 were predicted to be plantbeneficial bacteria with endophytic potential [58, 81, 82]. Also, M47T1 and M24T3 showed nematicidal potential against B. xylophilus [81, 82]. On the contrary, Feng et al. [82] report that $P$. fluorescens GcM5-1A is potentially plant pathogenic encoding a fully functional T3SS and 79 virulence factors. This study presents the complete genome analysis of the B. xylophilus-associated bacterium S. marcescens PWN146. Our analysis suggests that PWN146 may have endophytic competence, or at least be considered facultative/opportunistic endophyte, with potential to influence plant metabolism and hormonal pathways besides providing nutritional capacities [62]. We have demonstrated that PWN146 is able to colonize plant tissues, occupying the intercellular spaces, but that it is unable to kill C. elegans or B. xylophilus. Moreover, S. marcescens PWN146 seems to be a highly fitness and competitive bacterium in terms of resources as well as in bacterial and fungal antagonisms. Further investigation continues to be important to understand how bacteria are involved in PWD. Still, we have now more evidence to reinforce the theory that B. xylophilusassociated bacteria may have a plant origin $[10,58]$.

Acknowledgments The authors would like to thank Prof. John Jones (The James Hutton Institute) for advice on an earlier draft of this manuscript; Sonia Humphris, Jenny A. Morris, and Pete Hedley (The James Hutton Institute) for all the support given in Serratia sp. PWN146 sequencing. This work was supported by the JSPS KAKENHI Grant numbers P14394 (to CSLV) and 26450204 (to KH); the European Project REPHRAME - Development of improved methods for detection, control and eradication of pine wood nematode in support of EU Plant Health policy, European Union Seventh Framework Programme FP7-KBBE2010-4; and FEDER Funds through the Operational Programme for Competitiveness Factors - COMPETE and National Funds through FCT-Foundation for Science and Technology under the Strategic Project PEst-C/AGR/UI0115/2011.

\section{Compliance with Ethical Standards}

Conflict of Interest The authors declare that they have no conflict of interest.

Sequence Data The nucleotide sequence data reported is available in the EMBL database under the accession number ERS1151563.

\section{References}

1. Vicente C, Espada M, Vieira P, Mota M (2012) Pine Wilt Disease: a threat to European forestry. Eur J Plant Pathol 133:89-99

2. Jones JT, Moens M, Mota M, Li H, Kikuchi T (2008) Bursaphelenchus xylophilus: opportunities in comparative genomics and molecular host-parasite interactions. Mol Plant Pathol 9:357368

3. Futai K (2013) Pine wood nematode, Bursaphelenchus xylophilus. Annu Rev Phytopathol 51:61-83, 65

4. Zhao L, Mota M, Vieira P, Butcher RA, Sun J (2014) Interspecific communication between pinewood nematode, its insect vector, and associated microbes. Trends Parasitol 30:299-308

5. Zhao BGZ, Ang HLW, An SFH, An ZMH (2003) Distribution and pathogenicity of bacteria species carried by Bursaphelenchus xylophilus in China. Nematol 5:899-906

6. Zhao BG, Lin F (2005) Mutualistic symbiosis between Bursaphelenchus xylophilus and bacteria of the genus Pseudomonas. Forest Pathology 35:339-345

7. Vicente CSL, Ikuyo Y, Mota M, Hasegawa K (2013) Pinewood nematode-associated bacteria contribute to oxidative stress resistance of Bursaphelenchus xylophilus. BMC Microbiol 13:299

8. Cheng XY, Tian XL, Wang YS, Lin RM, Mao ZC, Chen N, Xie BY (2013) Metagenomic analysis of the pinewood nematode microbiome reveals a symbiotic relationship critical for xenobiotics degradation. Sci Rep 3:1-10

9. Paiva G, Proença DN, Francisco R et al (2013) Nematicidal bacteria associated to pinewood nematode produce extracellular proteases. PLoS ONE 8:e79705

10. Nascimento FX, Hasegawa K, Mota M, Vicente CSL (2015) Bacterial role in pine wilt disease development - review and future perspectives. Environ Microbiol Rep 7:51-63

11. Proença DN, Francisco RCV, Lopes A, Fonseca L, Abrantes IMO, Morais PV (2010) Diversity of bacteria associated with Bursaphelenchus xylophilus and other nematodes isolated from Pinus pinaster trees with pine wilt disease. PloS ONE 5:e15191

12. Vicente CSL, Nascimento F, Espada M, Mota M, Oliveira S (2011) Bacteria associated with the pinewood nematode Bursaphelenchus xylophilus collected in Portugal. Ant Van Leeuwenhoek 100:477481

13. Grimont F, Grimont P (2006) The genus Serratia. Prokaryotes 6: 219-244

14. Euzéby T (2015) List of Prokaryotic names with standing in nomenclature. Accessed in 2 of December 2015.

15. Iguchi A, Nagaya Y, Pradel E et al (2014) Genome evolution and plasticity of Serratia marcescens, an important multidrug-resistant nosocomial pathogen. Genome Biol Evol 6:2096-2110

16. Flyg C, Kenne K, Boman HG (1980) Insect pathogenic properties of Serratia marcescens: phage-resistant mutants with a decreased resistance to Cecropia immunity and a decreased virulence to Drosophila. J Gen Microbiol 120:173-181

17. Gillis A, Rodríguez M, Santana MA (2014) Serratia marcescens associated with bell pepper (Capsicum annuum L.) soft-rot disease under greenhouse conditions. Eur J Plant Pathol 138:1-8

18. Vicente CSL, Nascimento F, Espada M, Barbosa P, Mota M, Glick BR, Oliveira S (2012) Characterization of bacteria associated with pinewood Nematode Bursaphelenchus xylophilus. PLoS ONE 7: e46661

19. Brenner S (1974) The genetics of the nematode Caenorhabditis elegans. Genetics 77:71-94

20. Schneider CA, Rasband WS, Eliceiri KW (2012) NIH Image to ImageJ: 25 years of image analysis. Nature Methods 9:671-675

21. Takemoto S (2008) Population ecology of Bursaphelenchus xylophilus. In Pine Wilt Disease. Zhao BG, Futai K, Sutherland JR, Takeuchi Y. Kato Bunmeisha: Springer. pp. 105-122.

22. Barbosa PB, Ima ASL, Ieira PV, Ias LSD, Inoco MTT, Arroso JGB, Edro LGP (2010) Nematicidal activity of essential oils and volatiles derived from Portuguese aromatic flora against the pinewood nematode, Bursaphelenchus xylophilus. Nematol 42:8-16

23. Futai K, Furuno T (1979) The variety of resistances among pine species to pine wood nematode, Bursaphelenchus lignicolus. Bull Kyoto Univ For 51:23-36 
24. Fang ZD (1998) Methods in research of plant disease. Chinese Agricultural Publishing House, Beijing, China

25. Margulies M, Egholm M, Altman W et al (2005) Genome sequencing in microfabricated high-density picolitre reactors. Nature 437: 376-380

26. Chevreux B, Wetter T, Suhai S (1999) Genome sequence assembly using trace signals and additional sequence information. Computer Science and Biology: Proceedings of the German Conference on Bioinformatics (GCB) 99:45-56

27. Berlin K, Koren S, Chin CS, Drake JP, Landolin JM, Phillippy AM (2015) Assembling large genomes with single-molecule sequencing and locality- sensitive hashing. Nat Biotechnol 33:623-630

28. Chin CS, Alexander DH, Marks P et al (2013) Nonhybrid, finished microbial genome assemblies from long-read SMRT sequencing data. Nature Methods 10:563-569

29. Darling ACE, Mau B, Blattner FR, Perna NT (2004) Mauve: multiple alignment of conserved genomic sequence with rearrangements. Genome Res 14:1394-1403

30. Seeman T (2014) Prokka: rapid prokaryotic genome annotation. Bioinformatics 30:2068-2069

31. Conesa A, Gotz S, Garcia-Gomez JM, Terol J, Talon M, Robles M (2005) Blast2GO: a universal tool for annotation, visualization and analysis in functional genomics research. Bioinformatics 21:36743676

32. Moriya Y, Itoh M, Okuda S, Yoshizawa A, Kanehisa M (2007) KAAS: an automatic genome annotation and pathway reconstruction server. Nucleic Acids Res 35:W182-W185

33. Rutherford K, Parkhill J, Crook J, Horsnell T, Rice P, Rajandream MA, Barrell B (2000) Artemis: sequence visualization and annotation. Bioinformatics 16:944-945

34. Alikhan NF, Petty NK, Zakour NLB, Beatson SA (2011) BLAST Ring Image Generator (BRIG): simple prokaryote genome comparisons. BMC Genom 12:402

35. Tatusova T, Ciufo S, Fedorov B, O’Neill K, Tolstoy I (2014) RefSeq microbial genomes database: new representation and annotation strategy. Nuclei Acids Res 42:D553-D559

36. Aylward FO, Tremmel DM, Starrett GJ et al (2013) Complete genome of Serratia sp. strain associated with leaf-cutter ant fungus gardens. Genome Announc 1:e0023912

37. Chung WC, Chen LL, Lo WS, Kuo PA, Tu J, Kuo CH (2013) Complete genome sequence of Serratia marcescens WW4. Genome Announc 2:e00126123

38. Sandner-Miranda L, Vinuesa P, Soberón-Chávez G, MoralesEspinosa R (2016) Complete genome sequence of Serratia marcescens SmUNAM836, a nonpigmented multidrug-resistant strain isolated from a Mexican patient with obstructive pulmonary disease. Genome Announc 4:e1417-15

39. Dhillon BK, Laird MR, Shay JA et al (2015) IslandViewer 3: more flexible, interactive genomic island discovery, visualization and analysis. Nucleic Acids Res 43:W104-W108

40. Richter M, Rosselló-Móra R (2009) Shifting the genomic gold standard for the prokaryotic species definition. Proc Natl Acad Sci USA 106:19126-19131

41. Emms DM, Kelly S (2015) OrthoFinder: solving fundamental biases in whole genome comparisons dramatically improves orthogroup inference accuracy. Genome Biol 16:157

42. Gouy M, Guindon S, Gascuel O (2010) SeaView version 4: a multiplatform graphical user interface for sequence alignment and phylogenetic tree building. Mol Biol Evol 27:221-224

43. Tamura K, Stecher G, Peterson D, Filipski A, Kumar S (2013) MEGA6: Molecular Evolutionary Genetics Analysis version 6.0. Mol Biol Evol 1-12.

44. Shinya R, Morisaka H, Takeuchi Y, Ueda M, Futai K (2010) Comparison of the surface coat proteins of the pine wood nematode appeared during host pine infection and in vitro culture by a proteomic approach. Phytopathol 100:1289-1297
45. Shintani M, Sanchez ZK, Kimbara K (2015) Genomics of microbial plasmids classification and identification based on replication and transfer systems and host taxonomy. Front Microbiol 6:242

46. Smillie C, Garcillán-Barcia MP, Francia MV, Rocha EP, de la Cruz F (2010) Mobility of plasmids. Microbiol Mol Biol Rev 74:434 452

47. Hayes F (2003) Toxins-antitoxins: plasmid maintenance, programmed cell death, and cell cycle arrest. Science 301:1496-1499

48. Mitter B, Petric A, Shin MW, Chain PSG, Hauberg-Lotte L, Reinhold-Hurek B et al (2013) Comparative genome analysis of Burkholderia phytofirmans PsJN reveals a wide spectrum of endophytic lifestyles based on interaction strategies with host plants. Front Plant Sci 4:1-15

49. Darmon E, Leach DRF (2014) Bacterial genome instability. Microbiol Mol Biol Rev 78:1-39

50. Ternan NG, McGrath JW, McMullan G, Quinn JP (1998) Organophosphonates: occurrence, synthesis and biodegradation by microorganisms. World J Microbiol Biotechnol 14:635-647

51. Reith ME, Singh RK, Curtis B et al (2008) The genome of Aeromonas salmonicida subsp. salmonicida A449: insights into the evolution of a fish pathogen. BMC Genomics 9:427

52. Zhao BG, Lin F, Guo D, Li R, Li SN, Kulinich O, Ryss A (2009) Pathogenic roles of the bacteria carried by Bursaphelenchus mucronatus. J Nematol 41:11-16

53. Ali S, Duan J, Charles TC, Glick BR (2014) A bioinformatics approach to the determination of genes involved in endophytic behavior in Burkholderia spp. J Theor Biol 343:193-198

54. Wu X, Monchy S, Taghavi S, Zhu W, Ramos J, van der Lelie D (2010) Comparative genomics and functional analysis of nichespecific adaptation in Pseudomonas putida. FEMS Microbiol Rev 35:299-323

55. Couillault C, Ewbank JJ (2002) Diverse bacteria are pathogens of Caenorhabditis elegans. Infect Immun 70:4705-4707

56. Sato K, Yoshiga T, Hasegawa K (2014) Activated and inactivated immune responses in Caenorhabditis elegans against Photorhabdus luminescens TT01. SpringerPlus 3:274

57. Tripathi LP, Sowdhamini R (2008) Genome-wide survey of prokaryotic serine proteases: analysis of distribution and domain architectures of five serine protease families. BMC Genomics 9:549

58. Vicente CSL, Nascimento FX, Ikuyo Y, Cock PJA, Mota M, Hasegawa K (2016) The genome and genetics of a high oxidative stress tolerant Serratia sp. LCN16 isolated from the plant parasitic nematode Bursaphelenchus xylophilus. BMC Genomics 17:301

59. Berg G (2009) Plant-microbe interactions promoting plant growth and health: perspectives for controlled use of microorganisms in agriculture. Appl Microbiol Biotechnol 84:11-18

60. Taghavi S, Van der Lelie D, Hoffman A, Zhang YB, Walla MD, Vangronsveld J et al (2010) Genome sequence of the plant growth promoting endophytic bacterium Enterobacter $\mathrm{sp}$. 638. PLoS Genet 6:e1000943

61. Hardoim PR, van Overbeek LS, van Elsas JD (2008) Properties of bacterial endophytes and their proposed role in plant growth. Trends Microbiol 16:463-471

62. Friesen ML, Porter SS, Stark SC, Von Wettberg EJ, Sachs JL, Martinez-Romero E (2011) Microbially mediated plant functional traits. Annu Rev Ecol Evol Syst 42:23-46

63. Frank AC (2011) The genomes of endophytic bacteria. In Endophytes of Forest Trees: Biology and Applications. Pirttilä AM, Frank AC (eds). Springer Science+Business Media. pp. 107-136.

64. Baxter A, Mittler R, Suzuki N (2014) ROS as key players in plant stress signalling. J Exp Bot 65:1229-1240

65. Fones H, Preston GM (2012) Reactive oxygen and oxidative stress tolerance in plant pathogenic Pseudomonas. FEMS Microbiol Lett $327: 1-8$ 
66. Kim J, Park W (2014) Oxidative stress response in Pseudomonas putida. Appl Microbiol Biotechnol 98:6933-6946

67. Chiang SM, Schellhorn HE (2012) Regulators of oxidative stress response genes in Escherichia coli and their functional conservation in bacteria. Arch Biochem Biophys 525:161-169

68. Zeidler D, Zahringer U, Gerber I et al (2004) Innate immunity in Arabidopsis thaliana: lipopolysaccharides activate nitric oxide synthase (NOS) and induce defence genes. Proc Nat Acad Sci USA 44: 15811-15816

69. Li K, Chen WH, Bruner ST (2015) Structure and mechanism of siderophore-interacting protein from the fuscachelin gene cluster of Thermobifida fusca. Biochem 54:3989-4000

70. Taghavi S, Garafola C, Monchy S et al (2009) Genome survey and characterization of endophytic bacteria exhibiting a beneficial effect on growth and development of poplar trees. Appl Environ Microbiol 75:748-757

71. Martin VJJ, Yu Z, Mohn MW (1999) Recent advances in understanding resin acid biodegradation: microbial diversity and metabolism. Arch Microbiol 172:131-138

72. Adams AS, Aylward FO, Adams SM et al (2013) Mountain pine beetles colonizing historical and naïve host trees are associated with a bacterial community highly enriched in genes contributing to terpene metabolism. App Environ Microbiol 79:3468

73. Kende H (1993) Ethylene biosynthesis. Annu. Annu Rev Plant Physiol Plant Mol Biol 44:283-307

74. Ryu CM, Farag MA, Hu CH et al (2003) Bacterial volatiles promote growth in Arabidopsis. Proc Nat Acad Sci USA 100:49274932
75. Huang M, Oppermann-Sanio FB, Steinbuchel A (1999) Biochemical and molecular characterization of the Bacillus subtilis acetoin catabolic pathway. J Bacteriol 181:3837-3841

76. Choi O, Kim J, Kim JG et al (2008) Pyrroloquinoline quinone is a plant growth promotion factor produced by Pseudomonas fluorescens B16. Plant Physiol 146:657-668

77. Redondo-Nieto M, Barret M, Morrissey J et al (2013) Genome sequence reveals that Pseudomonas fluorescens F113 possesses a large and diverse array of systems for rhizosphere function and host interaction. BMC Genomics 14:54

78. Purushotham P, Sai Arun PVP, Prakash JSS, Podile AR (2012) Chitin binding proteins act synergistically with chitinases in Serratia proteamaculans 568. PloS ONE 7:e36714

79. Wang T, Si M, Song Y et al (2015) Type VI secretion system transports $\mathrm{Zn}^{2+}$ to combat multiple stresses and host immunity. PloS Pathogens 11:e1005020

80. Proença DN, Espírito-Santo C, Grass G, Morais PV (2012) Draft genome sequence of Pseudomonas sp. strain M47T1, carried by Bursaphelenchus xylophilus isolated from Pinus pinaster. J Bacteriol 194:4789-4790

81. Proença DN, Espírito-Santo C, Grass G, Morais PV (2012) Draft genome sequence of Serratia sp. strain M24T3, isolated from pinewood disease nematode Bursaphelenchus xylophilus. J Bacteriol 194:3764

82. Feng K, Li R, Chen Y, Zhao B, Yin T (2015) Sequencing and analysis of the Pseudomonas fluorescens GcM5-1A genome: a pathogen living in the surface coat of Bursaphelenchus xylophilus. PloS ONE 10:e141515 